\title{
Dynamics of electron injection and acceleration driven by laser wakefield in tailored density profiles
}

\author{
P. Lee, G. Maynard, T. L. Audet, and B. Cros ${ }^{\dagger}$ \\ LPGP, CNRS, Univ. Paris-Sud, Université Paris-Saclay, 91405 Orsay, France \\ R. Lehe and J.-L. Vay \\ Lawrence Berkeley National Laboratory, Berkeley, California 94720, USA
}

(Received 13 September 2016; published 16 November 2016)

\begin{abstract}
The dynamics of electron acceleration driven by laser wakefield is studied in detail using the particle-incell code WARP with the objective to generate high-quality electron bunches with narrow energy spread and small emittance, relevant for the electron injector of a multistage accelerator. Simulation results, using experimentally achievable parameters, show that electron bunches with an energy spread of $\sim 11 \%$ can be obtained by using an ionization-induced injection mechanism in a mm-scale length plasma. By controlling the focusing of a moderate laser power and tailoring the longitudinal plasma density profile, the electron injection beginning and end positions can be adjusted, while the electron energy can be finely tuned in the last acceleration section.
\end{abstract}

DOI: 10.1103/PhysRevAccelBeams.19.112802

\section{INTRODUCTION}

Laser wakefield acceleration capability to sustain fields in excess of $100 \mathrm{GV} / \mathrm{m}$ and produce short pulse electron bunches, makes it a promising way towards compact high energy accelerators for a wide range of applications. Multistage acceleration schemes [1] additionally have the potential to provide scalability and control, and are actively investigated for the development of future accelerators [2]. In these schemes, an optimized electron injector that produces a high quality electron beam with narrow energy spread and small emittance is one of the key issues.

The control of electron injection in the accelerating structures is an active research area [3]. Self-injection of plasma electrons into the accelerating structure occurs in the nonlinear regime of laser driven wakefield through plasma wave breaking [4-7] and depends on the coupled nonlinear evolution of laser pulse amplitude and plasma parameters. Control of electron injection can be achieved either by using an additional laser pulse as in the collidingpulse scheme [8] which consists in generating electrons in a selected region of the wakefield, or by shaping the plasma density, as for example in the density-transition based injection [9-13], which draws on a sharp downward plasma density transition between two adjacent regions of different densities to allow precise localized injection.

\footnotetext{
"patrick.lee@u-psud.fr

brigitte.cros@u-psud.fr
}

Published by the American Physical Society under the terms of the Creative Commons Attribution 3.0 License. Further distribution of this work must maintain attribution to the author $(s)$ and the published article's title, journal citation, and DOI.
Alternatively, the ionization-induced injection scheme [14-16] utilizing the large difference in ionization potentials between successive ionization states of trace atoms, allows one to create electrons at selected phases of the wakefield, resulting in low emittance beams. Experimentally, it can be achieved by focusing a single laser pulse in a gas medium composed of a mixture of high atomic number (Z) gas usually oxygen, nitrogen, or argon and low $\mathrm{Z}$ gas usually hydrogen or helium. The major drawback of this injection mechanism is that the produced electron beam exhibits a large energy spread. This is the case because this injection mechanism occurs continuously over the laser-plasma interaction region, as long as the laser intensity exceeds the ionization threshold, or up to the end of the mixed gas length, or until some competing mechanism, like beam loading, occurs. To reduce this wide energy spread, several experimental studies implement a mixed gas length reduced to a few $\mathrm{mm}$ in a two-stage laser wakefield accelerator configuration [17-22], the second accelerating stage acting as an energy filter; yet the generated electrons straight out of the injector still have a large energy spread, signifying that the mixed gas length is still longer than optimum and efficiency of coupling to the accelerating stage can be improved. As pointed out in [23], there is a linear correlation between the energy spread and the mixed gas length, implying that the beam quality can be improved by reducing the gas length. In this respect, many efforts were directed to tailoring the gas-density profile [24,25] and to using moderate power pulses [26] to limit the injection length, showing promising results.

Independent control of laser-wakefield acceleration and injection in two overlapped composite gas jets was recently demonstrated in [27] using self-injection and in [19] using 
ionization injection, resulting in tunable electron beams with reduced energy spread. In both cases, the obtained full-width at half-maximum (FWHM) energy spread of the produced electron bunch suggests that operating parameters can be further optimized.

We have performed numerical studies using the particlein-cell code WARP to determine optimized conditions for controlled ionization injection using a moderate power laser pulse, propagating in a single-stage mixed-gas cell. By analyzing the dynamics of electron injection and acceleration in this moderately nonlinear regime, we identify the mechanisms controlling the beginning and end of injection, and propose a way of tuning finely the electron beam energy while preserving its energy spread, by tailoring the longitudinal density profile of the last acceleration zone. This method produces electron bunches with a FWHM energy spread, $\Delta \mathcal{E}$ of $\sim 9 \mathrm{MeV}$ for a peak energy of $82.6 \mathrm{MeV}$.

The remainder of this paper is organized as follows: we present the simulation setup and the choice of laser-plasma parameters relevant to experimental conditions for an injector in Sec. II A. In Sec. II B, we describe the properties of the injected beam in the mixed-gas cell and give a detailed analysis of the dynamics of accelerated electrons. Finally, we discuss and illustrate in Sec. III the approach to tune the electron beam energy while preserving the energy spread.

\section{SIMULATION SETUP AND RESULTS}

\section{A. Choice of parameters}

Laser and plasma parameters are chosen in order to achieve electron acceleration to energies in the range of 50-200 MeV, well suited for an injector. The lower limit at $50 \mathrm{MeV}$ ensures that space charge effects will not be dominant, and that energy spread can be minimized as it scales as $1 / \gamma^{2}$, where $\gamma=\left[1-(v / c)^{2}\right]^{-1 / 2}$ is the Lorentz factor, $v$ the velocity of the electron and $c$ the speed of light. The upper limit is fixed at $200 \mathrm{MeV}$ to allow for a compact transport line for electron beam manipulation before coupling to the first accelerating structure. In addition, the electron bunch is required to have a small normalized transverse emittance of $\varepsilon_{n} \sim 0.1 \mathrm{~mm} \mathrm{mrad,} \mathrm{a} \mathrm{small} \mathrm{energy}$ spread (typically less than 10\%) and a large enough charge $(\geq 10 \mathrm{pC})$.

For the results reported in this paper, simulations were performed with WARP [28] using the azimuthal Fourier decomposition algorithm [29-31]. A field ionization module [32] based on the ADK model [33] was introduced in WARP to model ionization dynamics. A summary of the parameters used in our calculations is given in Table I. $a_{0}\left(z_{f}\right)$ is the maximum value of laser amplitude in normalized units, $a_{0}(z)=\max _{r, t}\left[e a(r, z, t) / m_{e} \omega c\right]$, where $\omega$ is the laser frequency, $e$ the electron charge, $m_{e}$ the electron mass and $a(r, z, t)$ the vector potential of the
TABLE I. List of parameters.

\begin{tabular}{|c|c|c|}
\hline $\begin{array}{l}\text { Maximum electron number } \\
\text { density on axis }\end{array}$ & $\max \left(n_{e 0}\right)$ & $7.8 \times 10^{18} \mathrm{~cm}^{-3}$ \\
\hline Longitudinal density profile & & ELISA profile \\
\hline Plasma length & $L_{p}$ & $2.4 \mathrm{~mm}$ \\
\hline Gas composition & & $99 \% \mathrm{H}_{2}+1 \% \mathrm{~N}_{2}$ \\
\hline Laser profile & & bi-Gaussian $^{\mathrm{a}}$ \\
\hline Normalized vector potential & $a_{0}\left(z_{f}\right)$ & 1.1 \\
\hline Laser wavelength & $\lambda_{0}$ & $0.8 \mu \mathrm{m}$ \\
\hline Laser spot size $\left(w^{\mathrm{b}}\right)$ & $\sigma$ & $17 \mu \mathrm{m}$ \\
\hline Laser duration (FWHM) & $\tau$ & $40 \mathrm{fs}$ \\
\hline Laser focal position & $z_{f}$ & $1.9 \mathrm{~mm}$ \\
\hline Laser polarization & & $\begin{array}{l}\text { linear (in the } \\
y \text { direction) }\end{array}$ \\
\hline Number of Fourier modes & & 2 \\
\hline Number of particles/cell & & 36 macro \\
\hline Cell size in $r$ & $\delta r$ & $\lambda_{0} / 2$ \\
\hline Cell size in $z$ & $\delta z$ & $\lambda_{0} / 50$ \\
\hline
\end{tabular}

${ }^{\mathrm{a}}$ Gaussian in temporal and spatial profiles.

${ }^{\mathrm{b}}$ Radius of the beam at $1 / e^{2}$.

initially bi-Gaussian laser pulse. The value of $a_{0}(z)=1.1$ corresponds to the maximum value of laser amplitude at the focal plane longitudinal position in vacuum, $z=z_{f}$.

The plasma electron density, $n_{e}$, is chosen to be in the range of $\left(10^{18}-10^{19}\right) \mathrm{cm}^{-3}$. In this range, the density is high enough for self-focusing of the laser pulse to be achieved, while low enough for the dephasing length $L_{d}$, $L_{d} \propto\left(\lambda_{p}^{3} / \lambda_{0}^{2}\right) a_{0} \propto n_{e 0}^{-3 / 2}$ (where a constant of order unity has been neglected), to be in the $\mathrm{mm}$ range and allow for electron acceleration to energies in the required range; here $\lambda_{p}$ is the plasma wavelength and $\lambda_{0}$ the laser wavelength. The density profile, so-called ELISA [34] profile, corresponds to the density profile achieved in a gas cell developed as an injector medium for multistage experiments planned in the frame of the CILEX project [35]. The ELISA profile was computed by 3D FLUID simulations performed using OPENFOAM [36], and characterized experimentally [34]. It is considered as the reference profile for the numerical studies presented here.

Figure 1 shows the evolution of $a_{0}$ (red solid line), the normalized vector potential of the laser pulse, and the plasma electron density normalized to its maximum (grey dashed line, ELISA profile), with respect to the propagation axis $z$. The shaded region of length $\sim 630 \mu$ m represents the window of electron injection in the laser wakefield structure. Four positions are marked: $z_{0}$ representing the beginning of electron injection, $z_{1}$ a position in the region between the beginning of electron injection and the position where $a_{0}$ reaches its maximum value $z_{2}$, and $z_{3}$ the end of electron injection. The laser pulse with moderate power, and normalized vector potential, $a_{0}$, is incident with a focus position in vacuum at $1900 \mu \mathrm{m}$, a position located in the down-ramp of ELISA profile. The reasons for using a moderate laser power are twofold: as can be seen in Fig. 1, 


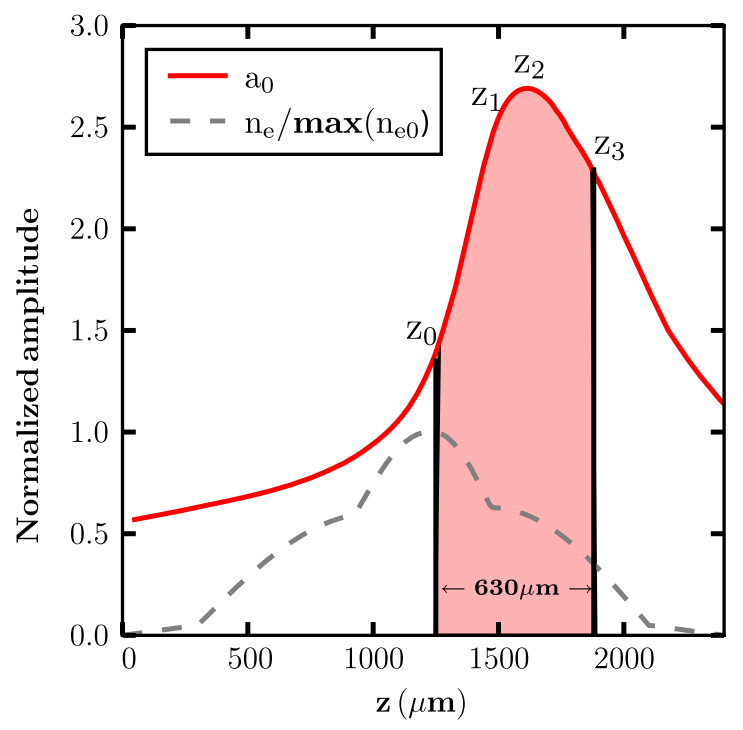

FIG. 1. Evolution of $a_{0}$ with respect to the propagation axis $z$. The grey dashed line shows the longitudinal density profile of the gas cell, or ELISA profile. The shaded area represents the injection range of length $\sim 630 \mu \mathrm{m}$. We define four markers in the injection zone: $z_{0}$, the position where injection begins; $z_{1}$, a position between $z_{0}$ and $z_{2} ; z_{2}$ the position where $a_{0}$ is maximum; $z_{3}$, the position where injection stops.

it leads to a slow growth of $a_{0}$ due to self-focusing of the laser pulse in the smooth up-ramp of density before reaching a maximum, thus delaying the trigger of the ionization-induced injection mechanism, and controlling the injection window to limit the energy spread; it prevents $a_{0}$ from reaching a value high enough for self-injection of electrons. In Ref. [15], a laser pulse with $a_{0} \sim 1.6$ was needed to ionize and inject the sixth electron of nitrogen and create an adequate wake potential to trap it, whereas self-trapping of electrons happens for $a_{0} \geq 4$. It is reported in [26] that low energy spread electron beams $(>120 \mathrm{MeV}$, $<15 \%$ ) were obtained via ionization-induced injection in a weakly relativistic laser wakefield induced by moderate power laser pulses (initial $a_{0}<1$ ).

\section{B. Electron beam properties}

A simulation with the parameters shown in Table I of Sec. II A was performed. In this section we discuss the properties of the resulting electron beam.

\section{Electron beam energy distribution}

The electron beam energy distribution is analyzed at the exit of the gas cell on the $z$ axis, $z_{\text {exit }}$, equal to the total plasma length: $z_{\text {exit }}=L_{\text {plasma }}=2.4 \mathrm{~mm}$. In Fig. 2 the charge density of the accelerated electron beam (black solid line) is plotted as a function of electron energy. In the simulation, all electrons are tagged and can be sorted according to their origin: the blue dashed line represents the charge density of electrons ionized from $\mathrm{N}^{5+} \rightarrow \mathrm{N}^{6+}$ and

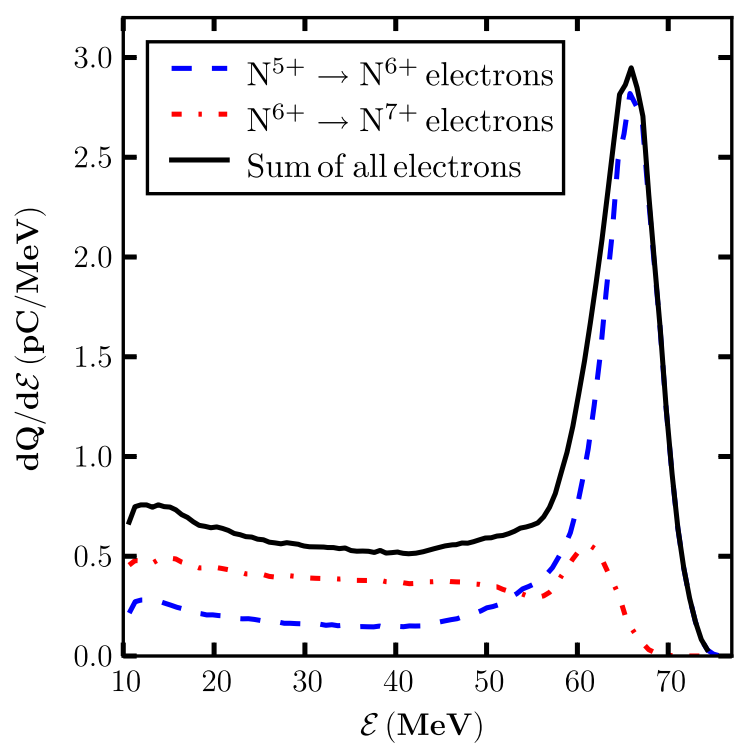

FIG. 2. The blue dashed line shows the energy spectrum of electrons from $\mathrm{N}^{5+} \rightarrow \mathrm{N}^{6+}$, whereas the red, dash-dotted line shows the energy spectrum of electrons from the ionization of $\mathrm{N}^{6+} \rightarrow \mathrm{N}^{7+}$. The black solid line represents the sum of the two spectra. Only $K$-shell electrons contribute to the electron beam energy spectrum at $z_{\text {exit }}$. Other electrons are not trapped but contribute to building the plasma wake. An energy cutoff at $10 \mathrm{MeV}$ is applied.

the red dash-dotted line represents the charge density of electrons ionized from $\mathrm{N}^{6+} \rightarrow \mathrm{N}^{7+}$. The energy distribution is shown for $\mathcal{E} \geq 10 \mathrm{MeV}$, corresponding to the minimum energy of trapped electrons.

This energy is linked to the structure of the generated wakefields, depending strongly on the interaction between the laser and the longitudinal density profile shown in Fig. 1. For an electron to be trapped in the wakefield, its Lorentz factor $\gamma$ is required to fulfill the condition [15]

$$
\Delta \Psi+1=\frac{\gamma}{\gamma_{\phi}^{2}},
$$

where $\Delta \Psi=e\left(\Psi_{f}-\Psi_{i}\right) /\left(m c^{2}\right), \gamma_{\phi}=\left(1-v_{\phi}^{2} / c^{2}\right)^{-1 / 2}$, and $v_{\phi}$ is the wake phase velocity. $\Psi$ is the wake potential and the subscripts $i$ and $f$ denote the initial and final trapping positions, respectively. Assuming all trapped electrons are ionized at the maximum of the laser envelope, $\Psi_{i}$ is then taken at the corresponding position. From this analysis, it is inferred that the trapped electrons have at least $\gamma \sim 20$ at the end of the injection phase. For this reason, the following analysis will focus on electrons with $\gamma \geq 20$.

As shown in Fig. 2, the electron spectrum is peaked at $65.7 \mathrm{MeV}$ with a FWHM energy spread, $\Delta \mathcal{E} / \mathcal{E}_{\text {peak }}=$ $13.1 \%$. The highest energy extends to $\sim 74 \mathrm{MeV}$. Only electrons initially in the K-shell of nitrogen are accelerated to higher energies as shown by the dashed blue line and red dash-dotted line. Other electrons coming either from 
nitrogen or from hydrogen are not trapped but contribute to building the plasma wake. This is in agreement with results obtained by other groups, for example with the 3D OSIRIS particle-in-cell code [15]. Note also that electrons coming from the heliumlike ion yield a higher charge and are the dominant contributors to the higher energy range of the energy spectrum, while those from the hydrogenlike ion yield a lower charge and are dominant contributors to the lower energy range.

\section{Dynamics of electron injection}

In order to analyze the dynamics of trapped electrons, we back-tracked 20000 randomly sampled trapped electrons $\left(10000\right.$ for $\mathrm{N}^{5+} \rightarrow \mathrm{N}^{6+}$ and 10000 for $\left.\mathrm{N}^{6+} \rightarrow \mathrm{N}^{7+}\right)$ beginning from $z_{\text {exit }}$ back to their ionization position, also corresponding to the position of their first appearance in the simulation. We then study the correlation between the energy of electrons at $z_{\text {exit }}$ and their position of ionization, as shown in Fig. 3.

In Fig. 3 is shown the energy of trapped electrons at $z_{\text {exit }}$ from (a) $\mathrm{N}^{5+} \rightarrow \mathrm{N}^{6+}$ and (b) $\mathrm{N}^{6+} \rightarrow \mathrm{N}^{7+}$. The trapped $K$ shell electrons are ionized in the range from 1250 to $1880 \mu \mathrm{m}$. Two kinds of electron distributions can be identified: Distribution I corresponds to electrons that have an energy higher than $\sim 55 \mathrm{MeV}$ and a position of ionization smaller that $z=1480 \mu \mathrm{m}$, while Distribution II corresponds to electrons with energy at $z_{\text {exit }}$ decreasing with respect to their position of ionization. Electrons coming from the heliumlike ion are ionized earlier in the propagation than those coming from the hydrogenlike ion, due to a lower ionization potential, 552 and $667 \mathrm{eV}$, respectively. The total charge in Distribution I is $49.4 \mathrm{pC}$, and $34.8 \mathrm{pC}$ in Distribution II, indicating that Distribution I represents $50.7 \%$ of the total number of trapped electrons.

Distribution I has a position of ionization between 1250 and $1480 \mu \mathrm{m}$ and an energy at $z_{\text {exit }}$ in the range of $55-74 \mathrm{MeV}$, where the spectrum is peaked as shown in Fig. 2. The line dividing the two distributions is located at $z=1480 \mu \mathrm{m}$, and corresponds to the position of the change of slope in the density down-ramp of the ELISA profile (cf. Fig. 1), indicating that the shape of the density profile has a major influence on the distribution of injected electrons.

For Distribution I, electrons from the heliumlike ion contribute a charge of $35.6 \mathrm{pC}$ while only $7.0 \mathrm{pC}$ is provided by electrons from the hydrogenlike ion. No obvious correlation between the ionization position and the electron energy at $z_{\text {exit }}$ is discerned for Distribution I, i.e., electrons that are ionized later in this interval can have the same energy as earlier ionized electrons, inferring that the injection and the acceleration processes are independent.

Distribution II starts at $z=1480 \mu \mathrm{m}$ and ends at $z=1880 \mu \mathrm{m}$. A clear correlation between the electron ionization position and electron energy at the exit of the
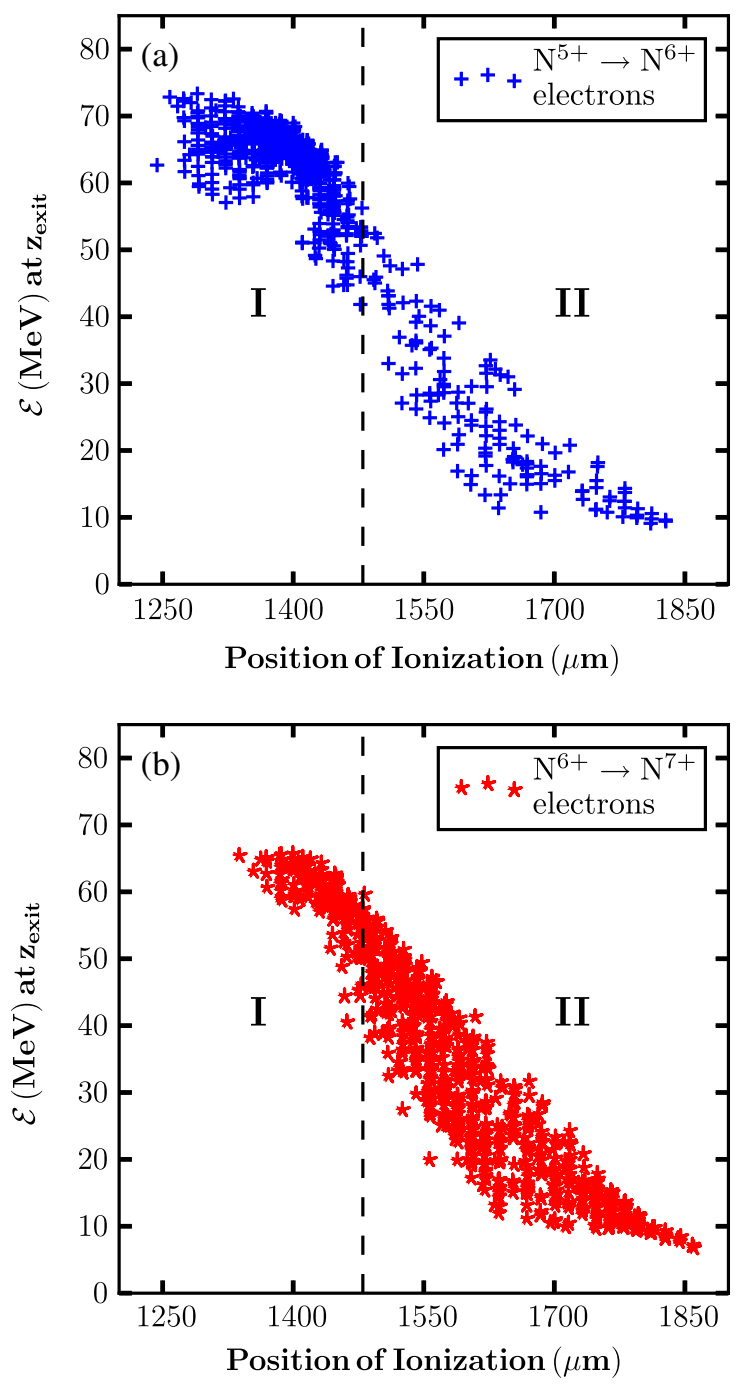

FIG. 3. Trapped $K$-shell electrons energy at $z_{\text {exit }}$ as a function of their ionization position; (a) blue crosses: electrons from $\mathrm{N}^{5+} \rightarrow \mathrm{N}^{6+}$, (b) red asterisks: electrons from $\mathrm{N}^{6+} \rightarrow \mathrm{N}^{7+}$. Two regions are marked in the distributions: Distribution I has energy larger than $55 \mathrm{MeV}$ and a position of ionization smaller than $z=1480 \mu \mathrm{m}$; Distribution II exhibits a decrease of energy for increased position of ionization.

plasma is observed, i.e., higher energy electrons are ionized first, implying continuous injection and acceleration of electrons. In this distribution, electrons from the hydrogenlike ion, as shown in Fig. 3(b), provide a charge of $27.8 \mathrm{pC}$ while electrons from the heliumlike ion provide a charge of $13.8 \mathrm{pC}$, as shown in Fig. 3(a).

\section{Dynamics of beam loading}

We further investigate the correlation between injection and acceleration processes by looking into the amplitude of accelerating wave structures.

In Fig. 4 are plotted the normalized laser field (blue/light grey), the normalized longitudinal wakefield (red/grey line) 

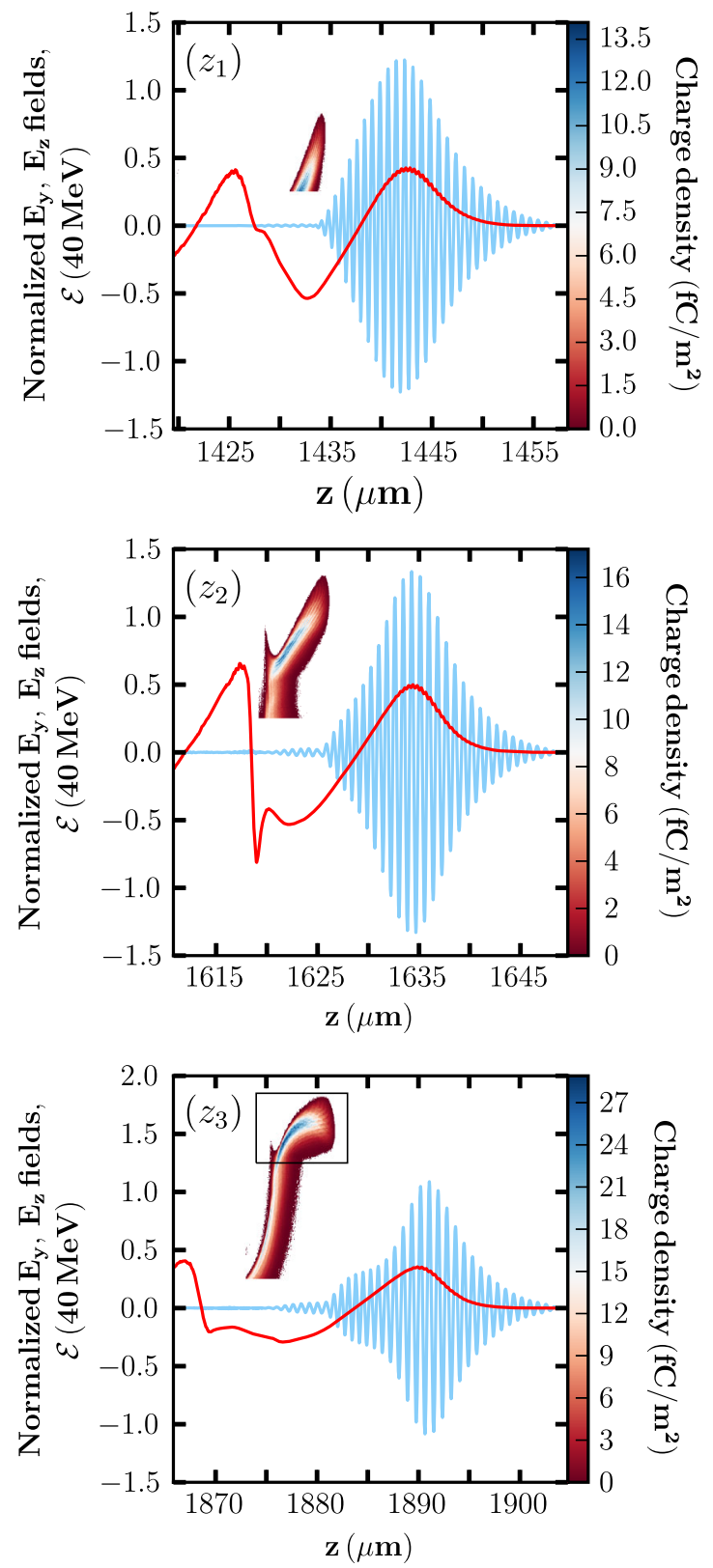

FIG. 4. Evolution of the normalized laser field, $e E_{y} / 2 m c \omega_{0}$ (in light blue/light gray), the normalized wakefield, $e E_{z} / m c \max \left(\omega_{p}\right)$ (in red/gray) and the energy, $\mathcal{E}$ of electrons (represented by a set of points) for the three positions of interest $z_{1-3}$ as marked in Fig. 1. The color bar represents charge density. The black rectangle at $z_{3}$ represents electrons in the high charge density region, with energy above $50 \mathrm{MeV}$.

and the energy of electrons divided by $40 \mathrm{MeV}$ (represented by a set of points with color scale for charge density) as a function of space around three positions in the density profile $z_{1}=1435 \mu \mathrm{m}, z_{2}$, and $z_{3}$ as marked in Fig. 1 . The laser propagates from left to right. Electrons that satisfy the trapping condition, given by Eq. (2.1) are trapped in the first bucket, defined by the region of negative $E_{z}$ bounded by zero crossing.
At $z_{1}$, the laser envelope is already deformed due to selffocusing, and the nonlinear accelerating wakefields are distorted due to the wakefield of injected electrons. Ionized electrons that satisfy the trapping condition are trapped and accelerated at the back of the bucket. However the widening of accelerating structures causes later trapped electrons to lag behind earlier injected ones; the latter are accelerated to a higher energy as compared to the former, an evidence of continuous injection of electrons in the bucket.

At the position of maximum laser intensity, $z_{2}$, we observe an increase in the charge density as compared to the previous position $z_{1}$, suggesting that more electrons are trapped in the bucket, and the wake is severely modified due to beam loading effects. Electrons at the back of the bucket experience a strong accelerating field, therefore their energy quickly catches up with previously injected electrons, consequently forming two high energy distributions. At the end of the ionization region, at $z_{3}$, the accelerating plasma wave structure is heavily beam loaded, resulting in the inhibition of further injection. The flattened normalized wakefield, $E_{s, N}=0.22$, giving $E_{s}=59.1 \mathrm{GV} / \mathrm{m}$, accelerates a rather energetic, homogenized electron bunch with a central energy of $\sim 62.6 \mathrm{MeV}$ in the highest charge density region, corresponding to the peak observed in the spectrum of Fig. 2.

Figure 5 shows a two-dimensional map in the $x-z$ plane of the electron density at position $z_{2}$. The laser amplitude is located between $z=1628 \mu \mathrm{m}$ and $z=1638 \mu \mathrm{m}$. A black dashed-line circle is superimposed to delimit the blown-out region. Trapped electrons are located in a region extending from the sheath of high density at the back of the cavity to the center of the blown-out region. The charge of the injected electron bunch in this structure is $Q=37.2 \mathrm{pC}$. This value can be compared to the analytical prediction for the amount of charge that can be loaded in the nonlinear wakes given in [37]. It can be evaluated as

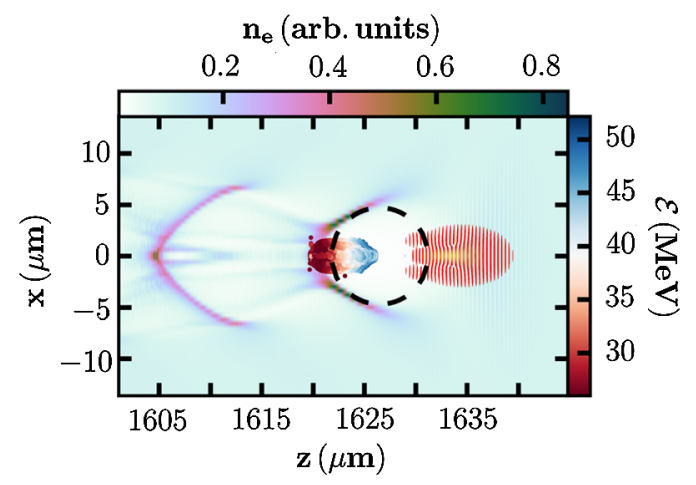

FIG. 5. Electron density in the $(x-z)$ plane at $z_{2}$, with superimposed laser amplitude and injected electron bunch. The horizontal color bar represents the normalized electron density in arbitrary unit and the vertical color bar depicts the energy of trapped electrons. A black dashed circle of $4.7 \mu \mathrm{m}$ radius is superimposed on the map to show the shape of the blown-out region. 


$$
Q_{s}=\frac{1}{4^{3}} \frac{1}{E_{s}}\left(k_{p} R_{b}\right)^{4}\left(\frac{m c^{2}}{r_{e}}\right)
$$

where $r_{e}=e^{2} /\left(m c^{2}\right)$ is the classical radius, $R_{b}$ is the radius of the blown-out region, $k_{p}$ is the wave number of the plasma waves and $E_{s}$ is the flattened wakefield amplitude. At $z_{2}$, the simulation gives $k_{p} R_{b}=1.74$ and $E_{s, N}=0.55$, giving $E_{s}=147.7 \mathrm{GV} / \mathrm{m}$. Using these values in Eq. (2.2), we obtain $Q_{s}=28.5 \mathrm{pC}$. This analytical prediction is of the same order of magnitude as the amount of charge calculated in the simulation, thus confirming that the operating regime is a beam-loaded blown-out regime.

At the end of the injection region, $z_{3}$, the high-energy electron bunch has a peak energy of $62.6 \mathrm{MeV}$ and a FWHM energy spread, $\Delta \mathcal{E} / \mathcal{E}_{\text {peak }}=14.2 \%$. Considering only high energy electrons in the energy range above $50 \mathrm{MeV}$, their charge $Q_{\text {high }}=43.6 \mathrm{pC}$ and they are distributed over a length, $\ell_{\text {bunch }}=6 \mu \mathrm{m}$. The charge of electrons with an energy of $\geq 10 \mathrm{MeV}$ at $z_{\text {exit }}$ is $Q_{\geq 10 \mathrm{MeV}}=$ $84.1 \mathrm{pC}$. The ratio of $Q_{\text {high }} / Q_{\geq 10 \mathrm{MeV}} \sim 0.52$, indicating that a significant amount of charge is found in the peak at $z_{3}$.

Figure 6 shows the evolution of the charge density with respect to the electron energy for three positions during the injection process. At $z_{1}$, the injection process has just begun, the spectrum exhibits a decrease of charge density with respect to electron energy, a characteristic of the continuous injection process. At $z_{2}$, a peak with a central energy of $32 \mathrm{MeV}$ is formed. At $z_{3}$, an increase of the population of electrons in the peak energy region is observed. Electrons injected earlier are now situated at the center of the bucket and form the bulk of the peak; they

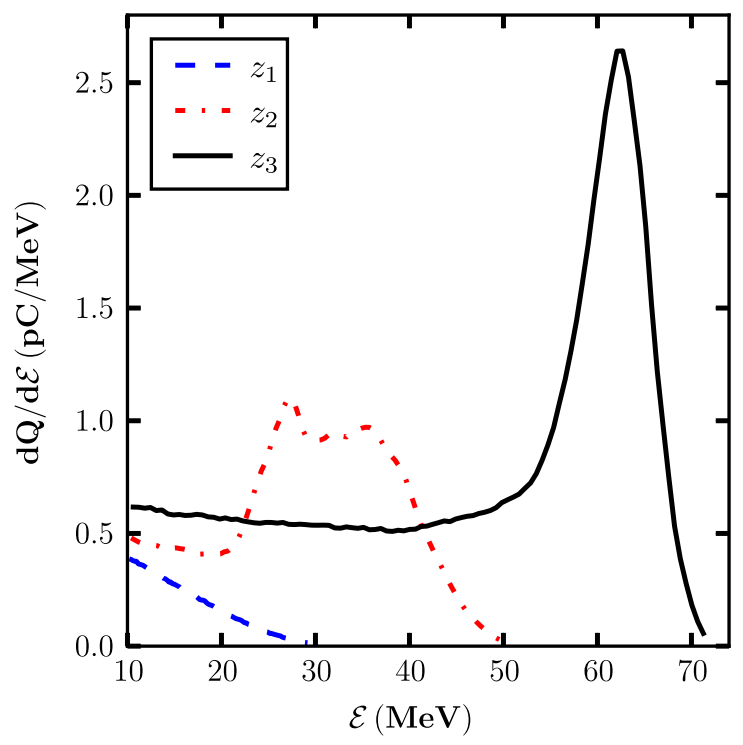

FIG. 6. Evolution of the charge density with respect to the energy with an energy cutoff at $10 \mathrm{MeV}$ at three different positions: $z_{1-3}$ corresponding to the cases of Fig. 4 . experience smaller accelerating wakefields compared to later injected electrons, some of which caught up with the initially injected ones and ended up populating the peak region.

At the exit of the gas cell, $z_{\text {exit }}$, the same electron bunch has increased its peak energy to $E_{\text {peak }}=65.7 \mathrm{MeV}$, and its FWHM energy spread is reduced to $\Delta E / E_{\text {peak }}=13.1 \%$. On one hand, the accelerating wakefields remain relatively flat throughout the length of the electron bunch up to the exit of the plasma gas cell, therefore the energy spread is preserved. On the other hand, due to the decrease in density along the propagation axis, the accelerating wakefields become weaker, so that the energy gained by the electron bunch between $z_{3}$ and $z_{\text {exit }}$ is small, $3.1 \mathrm{MeV}$.

The accelerating field, $E_{z}$, can be deduced directly with the equation $\Delta \mathcal{E}=q L_{\text {acc }} E_{z}$. The length over which most of acceleration occurs, $L_{\text {acc }}$ is the distance between the beginning and end of the position of ionization of the trapped electrons, respectively 1250 and $1880 \mu \mathrm{m}$ (cf. Fig. 3). For $\Delta \mathcal{E}=65.7 \mathrm{MeV}$ with $L_{\text {acc }}=630 \mu \mathrm{m}$, $E_{z}=104.3 \mathrm{GV} / \mathrm{m}$, which corresponds to the average field in the injection zone.

From the presented results, the fact that electrons with quite different trapping positions reach the same final energy can be explained as follows: first, a strong increase and the deformation of the accelerating fields occur during the trapping of electrons due to nonlinear effects, and play a significant role in homogenizing the energy of the initially trapped and later trapped electrons. Electrons are first trapped at the back of bucket, then the bucket enlarges due to laser self-focusing, as a result the newly generated electrons are trapped behind the earlier trapped electrons, where the accelerating field reaches a higher value; second, as soon as the trapping is suppressed, the high energy electron bunch, as long as it remains in the accelerating wakefields, is accelerated up to the exit of the gas cell.

\section{Beam emittance}

The beam emittance is a key parameter to determine the conditions to transport the beam to the second stage of the accelerator. Here we evaluate the normalized beam emittance along each axis as, $\varepsilon_{x_{i} \text {,rms }}$, using $\varepsilon_{x_{i} \text {,rms }}^{2}=\left\langle x_{i}^{2}\right\rangle\left\langle p_{i}^{2}\right\rangle-$ $\left\langle x_{i} p_{i}\right\rangle^{2}$ where $x_{i}$ are the positions, $p_{i}$ are the corresponding momenta normalized to $m_{e} c$. The emittance in $x$ and in $y$ directions are plotted as functions of electron energy in Figs. 7(a) and 7(b) respectively; the insets of Figs. 7(a) and 7 (b) show the distribution of electrons in $\left(x, p_{x}\right)$ and in $\left(y, p_{y}\right)$ phase space at the exit of the plasma, $z_{\text {exit }}$.

Considering all electrons with $\mathcal{E} \geq 10 \mathrm{MeV}$ in the first bucket, $\varepsilon_{x, \mathrm{rms}}=0.33 \mathrm{mmmrad}$ and $\varepsilon_{y, \mathrm{rms}}=2.09 \mathrm{~mm}$ mrad. $\varepsilon_{y, \text { rms }}$ is larger than $\varepsilon_{x, \text { rms }}$ because of the oscillation of electrons in the laser polarization $y$ direction. Defining the rms divergence as $\theta_{\perp}=\Delta p_{\perp, \mathrm{rms}} / p_{\|}$gives $\theta_{x}=6.9 \mathrm{mrad}$ and $\theta_{y}=18.5 \mathrm{mrad}$ at position $z_{\text {exit }}$. 

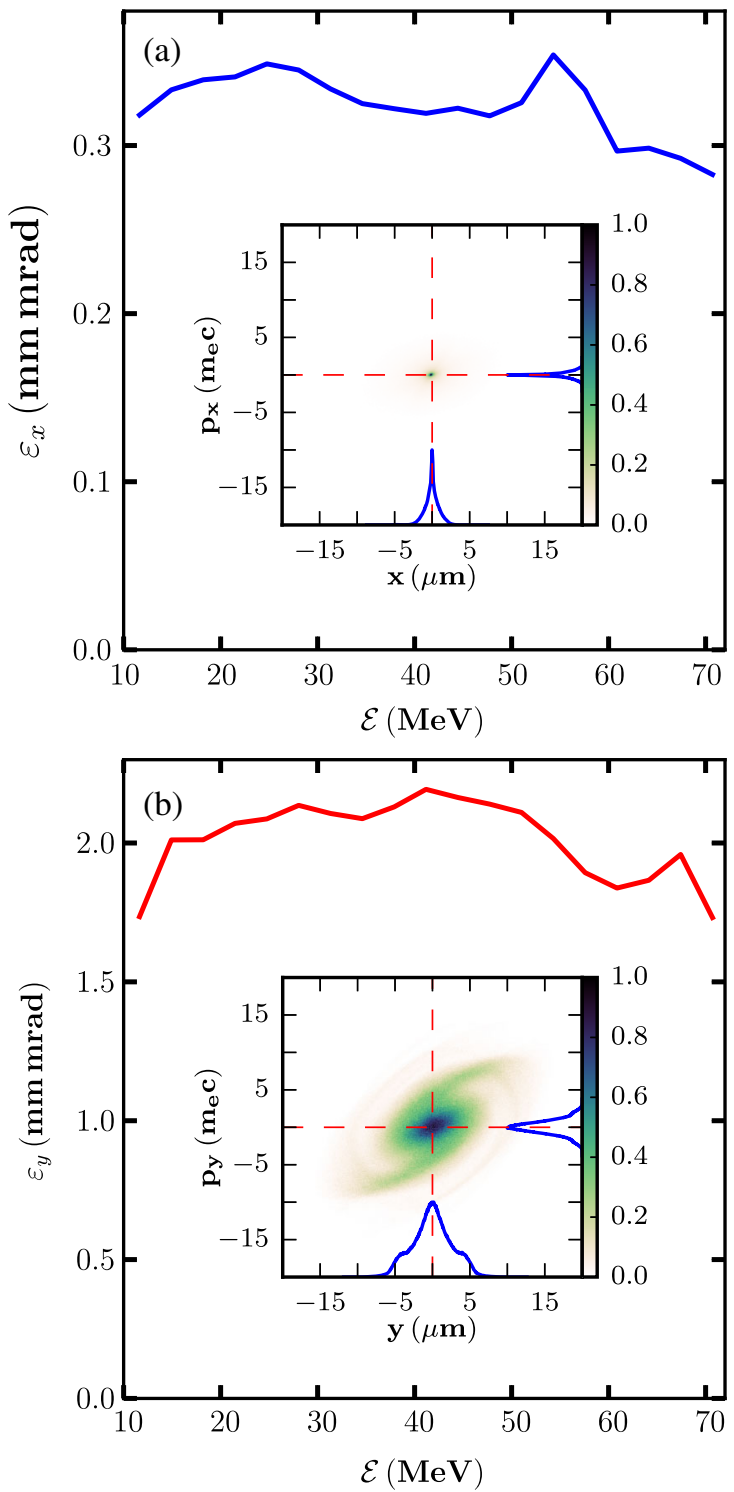

FIG. 7. Emittance of the electron bunch at the exit of the plasma, $z_{\text {exit }}$, as a function of electron energy in (a) $x$ and in (b) $y$ directions. The energy bin interval is $6.4 \mathrm{MeV}$. Insets of (a) and (b) represent the distribution of electrons with $\mathcal{E} \geq 10 \mathrm{MeV}$ in $\left(x, p_{x}\right)$ and in $\left(y, p_{y}\right)$ phase space. The color bars represent the electron density normalized to its maximum.

Figures 7(a) and 7(b) show that the emittance along the $x$ and $y$ axis are roughly constant with respect to electron energy, indicating that only the ionization process contributes to electron position $x_{i, \mathrm{rms}}$ and momentum $p_{i, \mathrm{rms}}$.

\section{TUNING ELECTRON BUNCH ENERGY WHILE PRESERVING ENERGY SPREAD}

Experimental results [19] in a two-stage gas target have shown that tailoring the density profile leads to the separation of the processes of electron injection and acceleration and permits independent control of both.
The results of Sec. II give indications on the ways to control injection and acceleration processes independently in a single gas target. In this section we explore the energy tunability of the electron beam with preservation of its energy spread.

Starting from the results obtained at $z_{3}$, the position where the injection stops for the ELISA profile, we tailor the density profile along the $z$-axis for $z>z_{3}$ in order to tune electron energy. The high energy part of the spectrum with $\mathcal{E} \geq 50 \mathrm{MeV}$ and the largest electron charge is selected at the end of the injection process $(z=1900 \mu \mathrm{m})$, as indicated by the black rectangle in Fig. $4\left(z_{3}\right)$. As pointed out in Sec. II, this electron bunch represents a significant portion of the total trapped electrons.

The strategy to maximize the energy gain of this electron bunch while preserving its energy spread is to achieve the largest possible, flat accelerating wakefield while maintaining the electron bunch in the acceleration phase. Numerical experiments were performed to further investigate this idea by tailoring the longitudinal density profile in the acceleration phase.

\section{A. Flat density profile beyond $z_{3}$}

A first example is illustrated in Fig. 8. The longitudinal density profile of interest is shown in Fig. 8(a). In Fig. 8(b) are plotted the electron bunch distribution together with the laser field and the wakefields at $z_{4}$, and at $z_{\text {exit }}$ in Fig. 8(c). Although electrons have gained $\sim 20 \mathrm{MeV}$ between $z_{3}$ and $z_{4}$, the accelerating wakefields are no longer flat, and electrons at the head and the tail of the bunch experience weaker accelerating wakefields as compared to the center part, resulting in the growth of energy spread in both these areas. As $a_{0}$ becomes $\sim 1$, the plasma wave is gradually becoming a regular sinusoidal oscillation with frequency $\omega_{p}(z)$. During the propagation between $z_{3}$ and $z_{4}$ or $z_{\text {exit }}$, the longitudinal extension of accelerating wakefields has shrinked significantly at what used to be the back of the bucket, and this effect caused the tail of the electron bunch to travel in decelerating wakefields; as a result, the tail of the bunch is being decelerated while the head is still being accelerated, resulting in an asymmetrical growth of the energy spread.

Figure 9 shows the spectra of accelerated electrons with energy $\mathcal{E} \geq 30 \mathrm{MeV}$ at different positions $z_{3}, z_{4}$ and $z_{\text {exit }}$. These spectra show that the electron bunch energy is increased, so as the charge at the peak energy between $z_{3}$ and $z_{4}$, thus improving the FWHM $\Delta \mathcal{E} / \mathcal{E}_{\text {peak }}$ to $11.5 \%$; however a decrease of $14.4 \%$ of the charge at the peak energy and an increase in the FWHM $\Delta \mathcal{E} / \mathcal{E}_{\text {peak }}$ to $12 \%$ for the spectrum at $z_{\text {exit }}$ results from the fact that some electrons are decelerated. This observation is explained by the shrinkage of the accelerating fields structure, leading to the subsequent slippage of electrons into the decelerating wakefields, as shown in Fig. 8(c). 
(a)

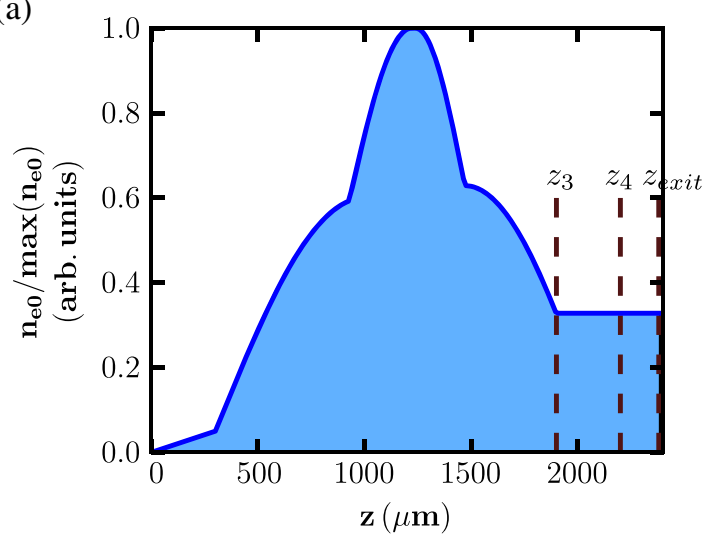

(b)

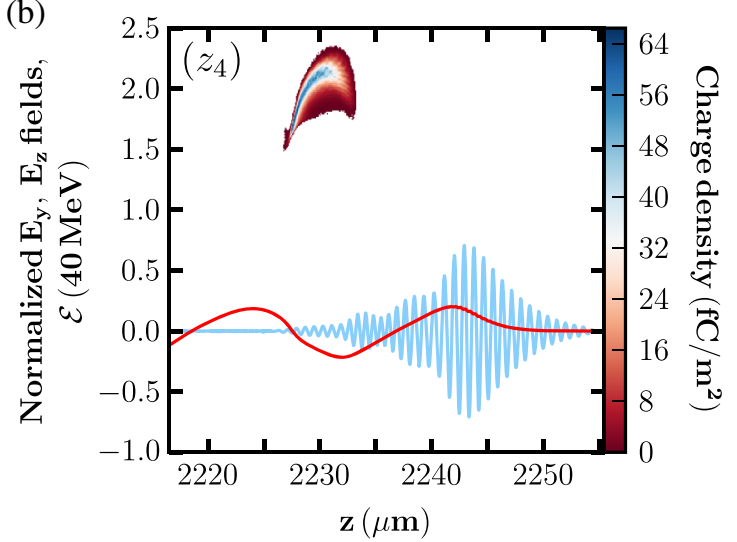

(c)

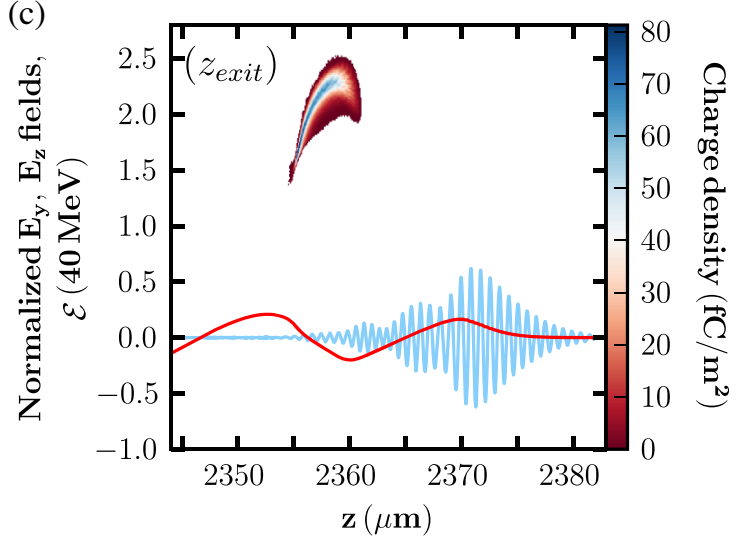

FIG. 8. (a) Tailored longitudinal density profile with a constant density extended from the end of the injection process. Three positions are marked, $z_{3}$, the end of the injection process; $z_{4}$, intermediate position between the end of the injection and the exit of the gas cell, $z_{\text {exit }}$. Two distinct instants $z_{4}$ and $z_{\text {exit }}$ of the normalized laser fields, $e E_{y} / 2 m c \omega_{0}$ (in light blue/light gray), the normalized wakefield, $e E_{z} / m c \max \left(\omega_{p}\right)$ (in red/gray) and the energy, $\mathcal{E}$, of traced electrons $\left(\mathcal{E} \geq 50 \mathrm{MeV}\right.$ at $\left.z_{3}\right)$ represented by a set of points, are shown in (b) and (c).

The evolution of the laser vector potential, $a_{0}$ for this case is similar to the one represented in Fig. 1, inferring that the variation in the density profile has no great influence on the laser propagation.

The energy gain starting from the end of the injection process $z_{3}$ up to the exit of the gas cell $z_{\text {exit }}$ is

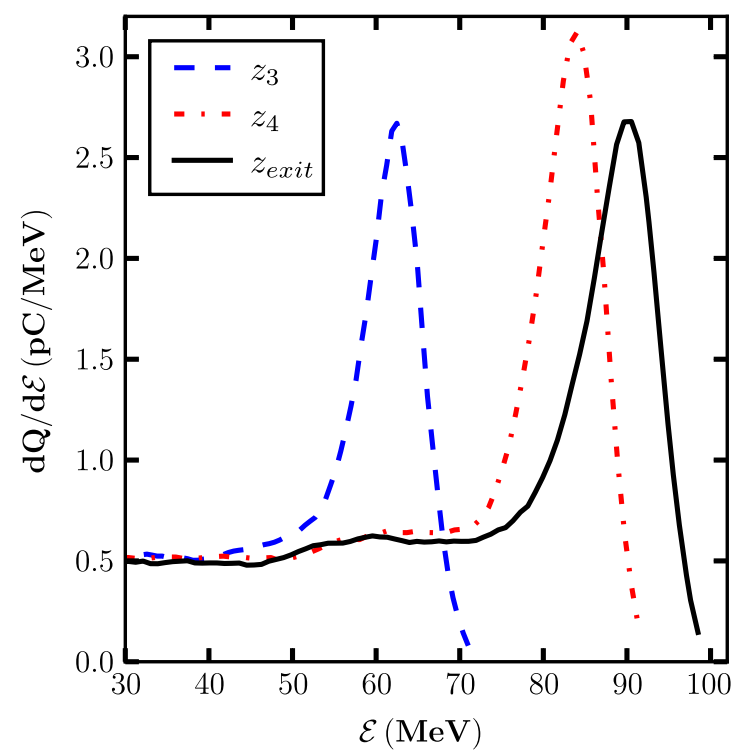

FIG. 9. Charge density of accelerated electrons having $\mathcal{E} \geq 30 \mathrm{MeV}$ with respect to electron energy obtained from the simulation using the longitudinal density profile featured in Fig. 8(a) at different positions $z_{3}, z_{4}$ and $z_{\text {exit }}$.

$\Delta \mathcal{E}=28.2 \mathrm{MeV}$, corresponding to an average accelerating field in the acceleration phase of $E_{z}=56.4 \mathrm{GV} / \mathrm{m}$.

In Fig. 10 are plotted the emittance along $x$ and $y$ directions with respect to electron energy, corresponding to the profile of Fig. 8(a). $\varepsilon_{x_{\text {rms }}}$ and $\varepsilon_{y_{\text {rms }}}$ are preserved, their values are comparable to those shown in Fig. 7. This result also confirms that there is no significant influence on the emittance caused by the interaction with the tail of the laser

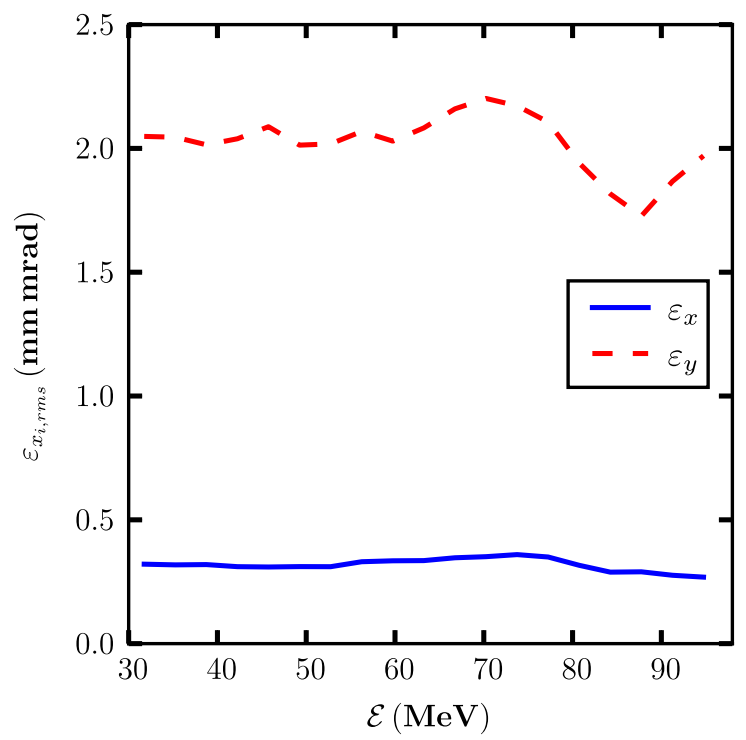

FIG. 10. Normalized beam emittances, $\varepsilon_{x_{\mathrm{rms}}}$ (blue solid line) and $\varepsilon_{y_{\mathrm{rms}}}$ (red dashed line) simulated with the longitudinal density profile in Fig. 8(a) with respect to energy. The energy bin interval is $6.8 \mathrm{MeV}$. 
pulse and the head of the electron beam, as observed in Fig. 8 [38].

\section{B. Linear density down-ramp beyond $z_{3}$}

The slippage of the tail of the electron bunch into the decelerating wakefields as shown in Fig. 8(c) leads to the growth of energy spread. Phase slippage in increasing density taper has been proposed [39-43] for controlling electron energy. Here, the decrease of longitudinal plasma density is used to minimize the growth of energy spread. In order to maintain the electron bunch in the plasma wave focusing and accelerating phase up to $z_{\text {exit }}$, the plasma wave extension has to be larger than the bunch extension i.e., $\lambda_{p}(z) / 4 \gtrsim \ell_{\text {bunch }}$. For $\ell_{\text {bunch }} \sim 6 \mu \mathrm{m}$, with $\lambda_{p}[\mu \mathrm{m}] \sim$ $3.3 \times 10^{10} \sqrt{n_{e 0}\left[\mathrm{~cm}^{-3}\right]}$, it gives $n_{e} \leq 1.94 \times 10^{18} \mathrm{~cm}^{-3}$. From Fig. 8(b) it can be observed that the plasma wave is approaching the linear regime and that the electron bunch begins to slip into the decelerating wakefields. We can therefore impose $n_{e}\left(z_{4}\right)=1.94 \times 10^{18} \mathrm{~cm}^{-3}$ and use a linear density gradient from $z_{3}$ as shown in Fig. 11(a).

In Figs. 11(b) and 11(c) are plotted the evolution of the electron bunch distribution, together with the laser fields and wakefields at two distinct positions $z_{4}$ and $z_{\text {exit }}$. The gradual decrease of density increases $\lambda_{p}$ and helps the electron bunch to stay in the accelerating phase of the wakefields; the symmetry of this electron bunch is preserved over a longer distance compared to the case with a flat density shown in Fig. 8. Also, due to the weaker accelerating wakefields as the density is decreased, the energy gain of the electron bunch is reduced, $\Delta \mathcal{E}=17 \mathrm{MeV}$, with an average accelerating field in the acceleration phase of $E_{z}=34 \mathrm{GV} / \mathrm{m}$.

Figure 12 depicts the evolution of the spectrum of the electron beam at $z_{3}, z_{4}$ and $z_{\text {exit }}$. Between $z_{3}$ and $z_{4}$, the energy of the electron bunch and the charge at the peak both increase, however the FWHM $\Delta \mathcal{E}$ is preserved. The comparison of spectra at $z_{4}$ and $z_{\text {exit }}$ shows that the peak energy is increased by $20 \mathrm{MeV}$, therefore FWHM $\Delta \mathcal{E} / \mathcal{E}_{\text {peak }}$ is reduced from $14.2 \%$ (at $z_{3}$ ) to $11.0 \%$ (at $z_{\text {exit }}$ ).

\section{Discussion}

The normalized beam emittances with respect to energy shown in Fig. 13 are very similar to those in Fig. 10. Using profiles in Fig. 8(a) and 11(a), $\varepsilon_{x_{\mathrm{mms}}}$ and $\varepsilon_{y_{\mathrm{rms}}}$ in both cases are preserved.

Figure 14 summarizes the energy distribution of the electron bunches in the peak for each of the three longitudinal density profiles. The final charge remains at $Q=43.6 \mathrm{pC}$ for all three simulations, implying that no electrons were lost during the acceleration process.

In this simulation, the evolution of the laser vector potential, $a_{0}$, remains similar to the one represented in Fig. 1. This suggests that the tailored density profile in this region has no great influence on the laser propagation.
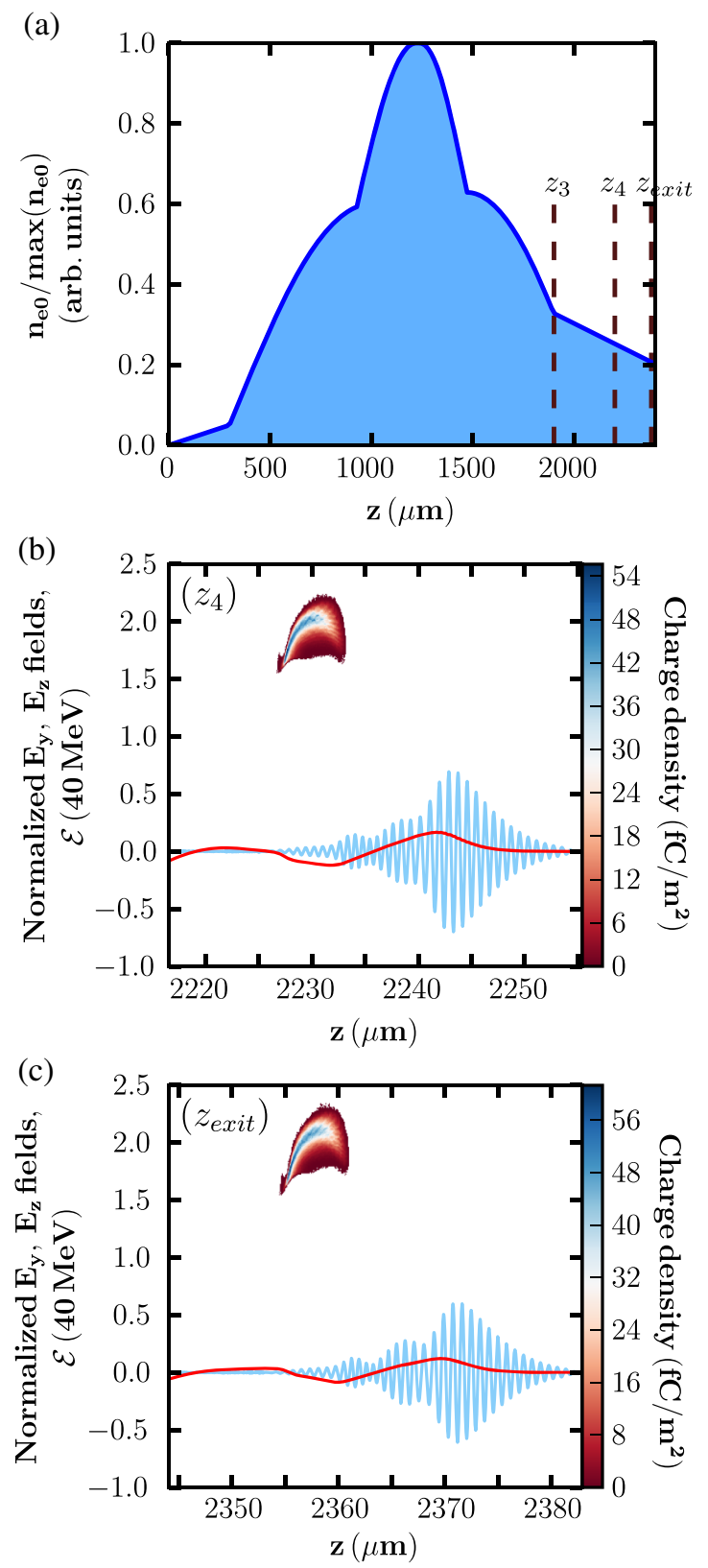

FIG. 11. (a) Tailored longitudinal density profile with a linear density down-ramp extended from the end of the injection process to the plasma exit. Three positions are marked, $z_{3}$, the end of the injection process; $z_{4}$, intermediate position between the end of the injection and the exit of the gas cell, $z_{\text {exit }}$. Two distinct instants $z_{4}$ and $z_{\text {exit }}$ of the normalized laser fields, $e E_{y} / 2 m c \omega_{0}$ (in light blue/light gray), the normalized wakefield, $e E_{z} / m c \max \left(\omega_{p}\right)$ (in $\mathrm{red} /$ gray) and the energy, $\mathcal{E}$ of traced electrons $(\mathcal{E} \geq 50 \mathrm{MeV}$ at $z_{3}$ ) represented by a set of points, are shown in (b) and (c).

Table II summarizes the values of peak energy and energy spread for the three cases. For the simulation with profile (a), $\mathcal{E}_{\text {peak }}$ at $z_{\text {exit }}$ is lower due to the decreasing accelerating wakefields in the descending phase of the density. The simulation with profile (c) gives the highest $\mathcal{E}_{\text {peak }}$ and the FWHM $\Delta \mathcal{E} / \mathcal{E}_{\text {peak }}$ at $z_{\text {exit }}$ is decreased to $12 \%$. The result that 


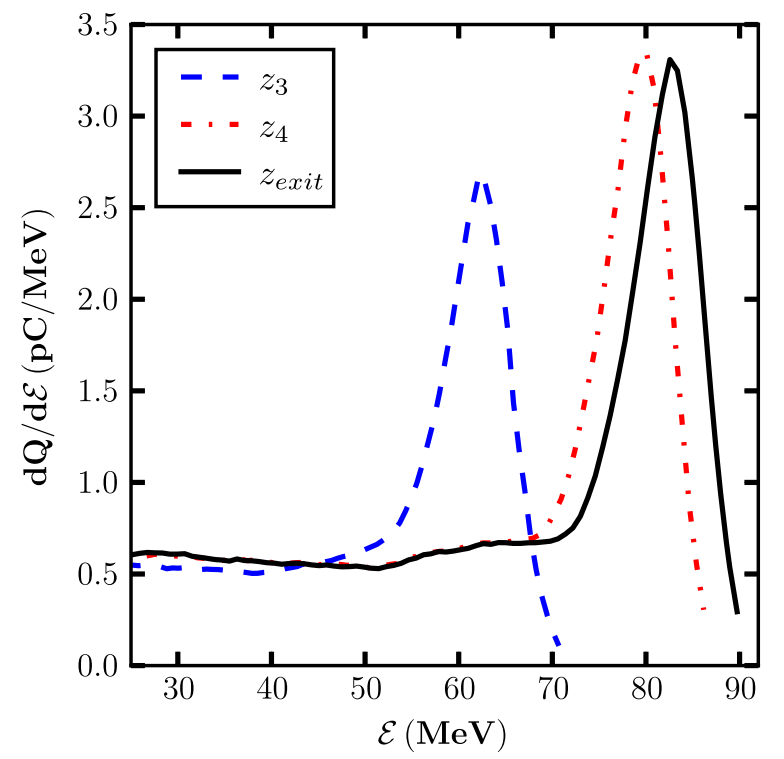

FIG. 12. Charge density of the accelerated electrons with respect to the electron energy simulated using the longitudinal density profile featured in Fig. 11(a) at different positions $z_{3}, z_{4}$ and $z_{\text {exit }}$.

offers the best compromise with the considered parameters is from the simulation with profile (b), the FWHM $\Delta \mathcal{E} / \mathcal{E}_{\text {peak }}$ is decreased to $11 \%$ and the $\mathcal{E}_{\text {peak }}$ is increased by $\sim 16.9 \mathrm{MeV}$ as compared to the result from the initial longitudinal density profile, depicted by profile (a).

From the presented results, the growth in FWHM $\Delta \mathcal{E} / \mathcal{E}_{\text {peak }}$ observed in Fig. 8(c) is mainly caused by the evolution from nonlinear, beam-loaded accelerating

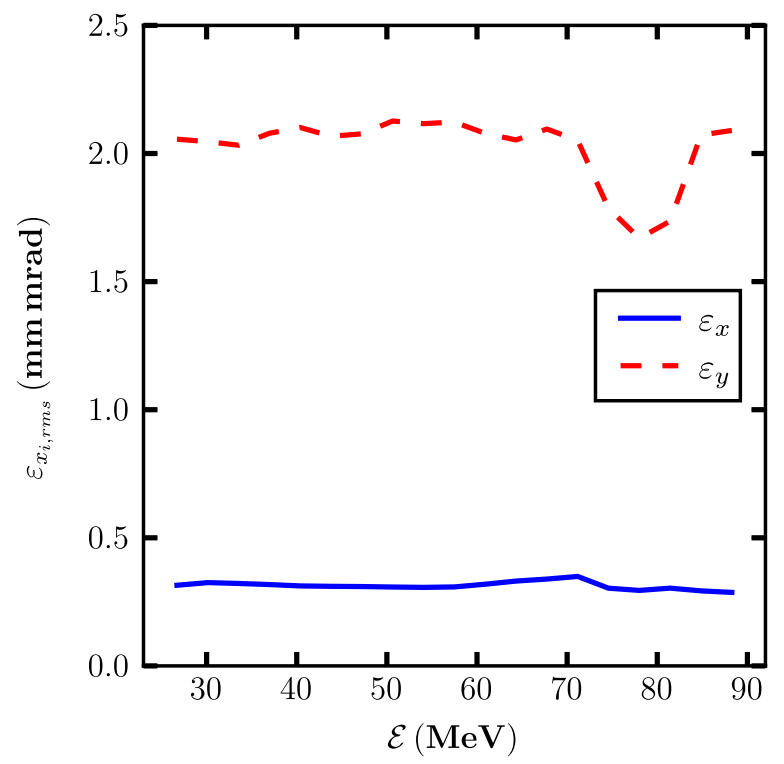

FIG. 13. Normalized beam emittances, $\varepsilon_{x, \text { rms }}$ (blue solid line) and $\varepsilon_{y, \text { rms }}$ (red dashed line) simulated with the longitudinal density profile in Fig. 11(a) with respect to energy. Only electrons of $\mathcal{E} \geq 25 \mathrm{MeV}$ are depicted. The energy bin interval is $6.7 \mathrm{MeV}$.

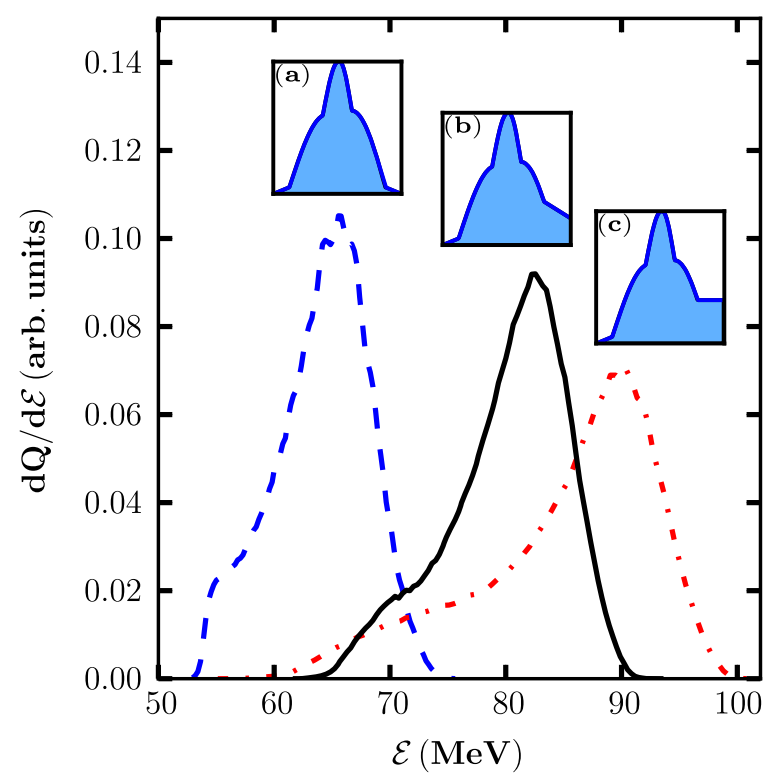

FIG. 14. Energy distribution of the traced electron bunch $\left(\mathcal{E} \geq 50 \mathrm{MeV}\right.$ at $\left.z_{3}\right)$ at the exit of the gas cell, $z_{\text {exit }}$, the onsets above each spectrum show the corresponding tailored longitudinal density profile: (a) with ELISA profile, (b) with a descending gradient, (c) with a plateau.

wakefields to sinusoidal oscillations when $a_{0}$ declines. This effect is mitigated using a descending gradient with the appropriate density predicted using the linear theory. Simulations with this longitudinal density profile show a decrease in the FWHM energy spread.

The presented method demonstrates a way to optimize the energy and the energy spread of electron bunches needed for injection into a multistage plasma-based accelerator. Other beam parameters should also be optimized before they could be used in high energy applications, such as the beam charge, to be increased by at least a factor of 2 , and the beam emittance, to be reduced to $1 \mathrm{~mm}$ mrad or less. Optimization of these two parameters while maintaining the energy spread is foreseen through tailoring of the driving laser beam distribution and is the goal of future work.

The obtained electron bunch properties are suitable for example for very high energy electron therapy (VHEET), which requires an energy range between 50 and $250 \mathrm{MeV}$ for treatment of deep-seated tumors $(>10 \mathrm{~cm})[44,45]$.

TABLE II. Comparison of the peak energy, $\mathcal{E}_{\text {peak }}$ and FWHM $\Delta \mathcal{E} / \mathcal{E}_{\text {peak }}$ of the accelerated electron bunches in different longitudinal density profile.

\begin{tabular}{lccc}
\hline \hline & $\begin{array}{c}\text { Longitudinal density } \\
\text { profile }\end{array}$ & $\begin{array}{c}\text { Peak energy, } \\
\mathcal{E}_{\text {peak }}(\mathrm{MeV})\end{array}$ & $\begin{array}{c}\text { FWHM } \\
\Delta \mathcal{E} / \mathcal{E}_{\text {peak }}(\%)\end{array}$ \\
\hline (a) & ELISA profile & 65.7 & 13.1 \\
(b) & Descending gradient & 82.6 & 11.0 \\
(c) & Plateau & 90.8 & 12.0 \\
\hline \hline
\end{tabular}




\section{CONCLUSION}

We have presented a detailed analysis of electron dynamics in the injection and acceleration processes. With the chosen laser plasma parameters, simulation results produce an electron bunch with $\mathcal{E}_{\text {peak }}$ of $65.7 \mathrm{MeV}$, a FWHM energy spread $\Delta \mathcal{E} / \mathcal{E}_{\text {peak }}$ of $13.1 \%$ and a charge of $43.6 \mathrm{pC}$, where the FWHM energy spread is yet to be improved. The moderate power laser pulse restricts the injection to only ionization induced injection and a focal position in the descending gradient of the longitudinal density profile allows a slow growth of the vector potential, $a_{0}$, delaying the ionization processes, resulting in the shortening of the injection range as compared to the plasma length. In this parameter range, beam loading effects are responsible for two distinct phenomena: the inhibition of the injection process and the homogenization of the energy distribution of the trapped electron bunch. By separating injection and acceleration processes, an additional degree of control is gained in the acceleration process. We tailored the longitudinal density profile starting from the position of the end of the injection process up to the end of the plasma, in order to accelerate the electron bunch to a higher energy while preserving its energy spread.

Results from WARP simulations using three Fourier modes in the azimuthal Fourier decomposition algorithm show no significant modification in the beam properties, confirming the accuracy of simulations using two Fourier modes, as presented in this article. The best possible result with the considered parameters is obtained using the descending gradient in the longitudinal density profile. This approach takes into consideration the maximization of the accelerating wakefields and the rephasing of the electron bunch to minimize the FWHM energy spread. It is shown that both the charge and the emittance in $x$ and $y$ directions of the electron bunch are preserved and the FWHM $\Delta \mathcal{E} / \mathcal{E}_{\text {peak }}$ is reduced.

\section{ACKNOWLEDGMENTS}

We are thankful to Carlo Benedetti for insightful discussion. We acknowledge the use of the computing facility cluster Grappe Massivement Parallèle de Calcul Scientifique (GMPCS) of the LUMAT federation (FR LUMAT 2764) and the partial financial support of the Laboratoire d'Excellence Physique: Atomes Lumière Matière, within the Model_LPA project. This work was supported partially by the Director, Office of Science, of the U.S. Department of Energy under Contract No. DE-AC02$05 \mathrm{CH} 11231$.

[1] W. Leemans and E. Esarey, Laser-driven plasma-wave electron accelerators, Phys. Today 62, No. 2, 44 (2009).

[2] Compact European plasma accelerator with superior beam quality, http://www.eupraxia-project.eu/.
[3] V. Malka, Laser plasma accelerators, Phys. Plasmas 19, 055501 (2012).

[4] J. Faure, C. Rechatin, A. Norlin, A. Lifschitz, Y. Glinec, and V. Malka, Controlled injection and acceleration of electrons in plasma wakefields by colliding laser pulses, Nature (London) 444, 737 (2006).

[5] C. G. R. Geddes, C. Toth, J. van Tilborg, E. Esarey, C. B. Schroeder, D. Bruhwiler, C. Nieter, J. Cary, and W. P. Leemans, High-quality electron beams from a laser wakefield accelerator using plasma-channel guiding, Nature (London) 431, 538 (2004).

[6] S. P. D. Mangles, C. D. Murphy, Z. Najmudin, A. G. R. Thomas, J. L. Collier, A. E. Dangor, E. J. Divall, P. S. Foster, J. G. Gallacher, C. J. Hooker, D. A. Jaroszynski, A. J. Langley, W. B. Mori, P. A. Norreys, F. S. Tsung, R. Viskup, B. R. Walton, and K. Krushelnick, Monoenergetic beams of relativistic electrons from intense laser-plasma interactions, Nature (London) 431, 535 (2004).

[7] S. Y. Kalmykov, A. Beck, S. A. Yi, V. N. Khudik, M. C. Downer, E. Lefebvre, B. A. Shadwick, and D. P. Umstadter, Electron self-injection into an evolving plasma bubble: Quasi-monoenergetic laser-plasma acceleration in the blowout regime, Phys. Plasmas 18, 056704 (2011).

[8] E. Esarey, R. F. Hubbard, W. P. Leemans, A. Ting, and P. Sprangle, Electron Injection into Plasma Wakefields by Colliding Laser Pulses, Phys. Rev. Lett. 79, 2682 (1997).

[9] S. Fourmaux, K. T. Phuoc, P. Lassonde, S. Corde, G. Lebrun, V. Malka, A. Rousse, and J. C. Kieffer, Quasimonoenergetic electron beams production in a sharp density transition, Appl. Phys. Lett. 101, 111106 (2012).

[10] P. Brijesh, C. Thaury, K. T. Phuoc, S. Corde, G. Lambert, V. Malka, S. P. D. Mangles, M. Bloom, and S. Kneip, Tapered plasma channels to phase-lock accelerating and focusing forces in laser-plasma accelerators, Phys. Plasmas 19, 063104 (2012).

[11] M. Burza, A. Gonoskov, K. Svensson, F. Wojda, A. Persson, M. Hansson, G. Genoud, M. Marklund, C.-G. Wahlstrm, and O. Lundh, Laser wakefield acceleration using wire produced double density ramps, Phys. Rev. ST Accel. Beams 16, 011301 (2013).

[12] A. Buck, J. Wenz, J. Xu, K. Khrennikov, K. Schmid, M. Heigoldt, J. M. Mikhailova, M. Geissler, B. Shen, F. Krausz, S. Karsch, and L. Veisz, Shock-Front Injector for High-Quality Laser-Plasma Acceleration, Phys. Rev. Lett. 110, 185006 (2013).

[13] K. Schmid, A. Buck, C. M. S. Sears, J. M. Mikhailova, R. Tautz, D. Herrmann, M. Geissler, F. Krausz, and L. Veisz, Density-transition based electron injector for laser driven wakefield accelerators, Phys. Rev. ST Accel. Beams 13, 091301 (2010).

[14] C. E. Clayton, J. E. Ralph, F. Albert, R. A. Fonseca, S. H. Glenzer, C. Joshi, W. Lu, K. A. Marsh, S. F. Martins, W. B. Mori, A. Pak, F. S. Tsung, B. B. Pollock, J. S. Ross, L. O. Silva, and D. H. Froula, Self-Guided Laser Wakefield Acceleration beyond $1 \mathrm{GeV}$ Using Ionization-Induced Injection, Phys. Rev. Lett. 105, 105003 (2010).

[15] A. Pak, K. A. Marsh, S. F. Martins, W. Lu, W. B. Mori, and C. Joshi, Injection and Trapping of Tunnel-Ionized 
Electrons into Laser-Produced Wakes, Phys. Rev. Lett. 104, 025003 (2010).

[16] C. McGuffey, A. G. R. Thomas, W. Schumaker, T. Matsuoka, V. Chvykov, F. J. Dollar, G. Kalintchenko, V. Yanovsky, A. Maksimchuk, K. Krushelnick, V. Y. Bychenkov, I. V. Glazyrin, and A. V. Karpeev, Ionization Induced Trapping in a Laser Wakefield Accelerator, Phys. Rev. Lett. 104, 025004 (2010).

[17] A. J. Gonsalves, K. Nakamura, C. Lin, D. Panasenko, S. Shiraishi, T. Sokollik, C. Benedetti, C. B. Schroeder, C. G. R. Geddes, J. van Tilborg, J. Osterhoff, E. Esarey, C. Toth, and W. P. Leemans, Tunable laser plasma accelerator based on longitudinal density tailoring, Nat. Phys. 7, 862 (2011).

[18] W. Wang, W. Li, J. Liu, C. Wang, Q. Chen, Z. Zhang, R. Qi, Y. Leng, X. Liang, Y. Liu, X. Lu, C. Wang, R. Li, and $\mathrm{Z}$. $\mathrm{Xu}$, Control of seeding phase for a cascaded laser wakefield accelerator with gradient injection, Appl. Phys. Lett. 103, 243501 (2013).

[19] G. Golovin, S. Chen, N. Powers, C. Liu, S. Banerjee, J. Zhang, M. Zeng, Z. Sheng, and D. Umstadter, Tunable monoenergetic electron beams from independently controllable laser-wakefield acceleration and injection, Phys. Rev. ST Accel. Beams 18, 011301 (2015).

[20] J. S. Liu, C. Q. Xia, W. T. Wang, H. Y. Lu, C. Wang, A. H. Deng, W. T. Li, H. Zhang, X. Y. Liang, Y. X. Leng, X. M. Lu, C. Wang, J. Z. Wang, K. Nakajima, R. X. Li, and Z. Z. $\mathrm{Xu}$, All-Optical Cascaded Laser Wakefield Accelerator Using Ionization-Induced Injection, Phys. Rev. Lett. 107, 035001 (2011).

[21] H. T. Kim, K. H. Pae, H. J. Cha, I. J. Kim, T. J. Yu, J. H. Sung, S. K. Lee, T. M. Jeong, and J. Lee, Enhancement of Electron Energy to the Multi-GeV Regime by a Dual-Stage Laser-Wakefield Accelerator Pumped by Petawatt Laser Pulses, Phys. Rev. Lett. 111, 165002 (2013).

[22] B. B. Pollock, C. E. Clayton, J. E. Ralph, F. Albert, A. Davidson, L. Divol, C. Filip, S. H. Glenzer, K. Herpoldt, W. Lu, K. A. Marsh, J. Meinecke, W. B. Mori, A. Pak, T. C. Rensink, J. S. Ross, J. Shaw, G. R. Tynan, C. Joshi, and D. H. Froula, Demonstration of a Narrow Energy Spread, $\sim 0.5 \mathrm{GeV}$ Electron Beam from a Two-Stage Laser Wakefield Accelerator, Phys. Rev. Lett. 107, 045001 (2011).

[23] M. Chen, E. Esarey, C. B. Schroeder, C. G. R. Geddes, and W. P. Leemans, Theory of ionization-induced trapping in laser-plasma accelerators, Phys. Plasmas 19, 033101 (2012).

[24] M. Zeng, N. A. M. Hafz, K. Nakajima, L.-M. Chen, W. Lu, W. B. Mori, Z.-M. Sheng, and J. Zhang, Controlled ionization-induced injection by tailoring the gas-density profile in laser wakefield acceleration, J. Plasma Phys. 78, 363 (2012).

[25] C. Thaury, E. Guillaume, A. Lifschitz, K. T. Phuoc, M. Hansson, G. Grittani, J. Gautier, J.-P. Goddet, A. Tafzi, O. Lundh, and V. Malka, Shock assisted ionization injection in laser-plasma accelerators, Sci. Rep. 5, 16310 (2015).

[26] C. Kamperidis, V. Dimitriou, S. P. D. Mangles, A. E. Dangor, and Z. Najmudin, Low energy spread electron beams from ionization injection in a weakly relativistic laser wakefield accelerator, Plasma Phys. Controlled Fusion 56, 084007 (2014).

[27] M. Hansson, B. Aurand, X. Davoine, H. Ekerfelt, K. Svensson, A. Persson, C.-G. Wahlstrm, and O. Lundh, Down-ramp injection and independently controlled acceleration of electrons in a tailored laser wakefield accelerator, Phys. Rev. ST Accel. Beams 18, 071303 (2015).

[28] J.-L. Vay, D. P. Grote, R. H. Cohen, and A. Friedman, Novel methods in the particle-in-cell accelerator codeframework warp, Comput. Sci. Discovery 5, 014019 (2012).

[29] A. Lifschitz, X. Davoine, E. Lefebvre, J. Faure, C. Rechatin, and V. Malka, Particle-in-Cell modelling of laser-plasma interaction using Fourier decomposition, J. Comput. Phys. 228, 1803 (2009).

[30] A. Davidson, A. Tableman, W. An, F. Tsung, W. Lu, J. Vieira, R. Fonseca, L. Silva, and W. Mori, Implementation of a hybrid particle code with a PIC description in $r-z$ and a gridless description in $\phi$ into OSIRIS, J. Comput. Phys. 281, 1063 (2015).

[31] P. Lee, T. Audet, R. Lehe, J.-L. Vay, G. Maynard, and B. Cros, Modeling laser-driven electron acceleration using WARP with Fourier decomposition, Nucl. Instrum. Methods Phys. Res., Sect. A 829, 358 (2015).

[32] F. G. Desforges, B. S. Paradkar, M. Hansson, J. Ju, L. Senje, T. L. Audet, A. Persson, S. Dobosz-Dufrénoy, O. Lundh, G. Maynard, P. Monot, J.-L. Vay, C.-G. Wahlstrm, and B. Cros, Dynamics of ionization-induced electron injection in the high density regime of laser wakefield acceleration, Phys. Plasmas 21, 120703 (2014).

[33] M. Ammosov, N. Delone, and V. Krainov, Tunnel ionization of complex atoms and of atomic ions in an alternating electric field, Sov. Phys. JETP 64, 1191 (1986).

[34] T. Audet, F. Desforges, A. Maitrallain, S. D. Dufrénoy, M. Bougeard, G. Maynard, P. Lee, M. Hansson, B. Aurand, A. Persson, I. G. González, P. Monot, C.-G. Wahlstrm, O. Lundh, and B. Cros, Electron injector for compact staged high energy accelerator, Nucl. Instrum. Methods Phys. Res., Sect. A 829, 304 (2016).

[35] B. Cros et al., Laser plasma acceleration of electrons with multi-PW laser beams in the frame of CILEX, Nucl. Instrum. Methods Phys. Res., Sect. A 740, 27 (2014).

[36] H. G. Weller, G. Tabor, H. Jasak, and C. Fureby, A tensorial approach to computational continuum mechanics using object-oriented techniques, Comput. Phys. 12, 620 (1998).

[37] M. Tzoufras, W. Lu, F. S. Tsung, C. Huang, W. B. Mori, T. Katsouleas, J. Vieira, R. A. Fonseca, and L. O. Silva, Beam Loading in the Nonlinear Regime of Plasma-Based Acceleration, Phys. Rev. Lett. 101, 145002 (2008).

[38] R. Lehe, C. Thaury, E. Guillaume, A. Lifschitz, and V. Malka, Laser-plasma lens for laser-wakefield accelerators, Phys. Rev. ST Accel. Beams 17, 121301 (2014).

[39] T. Katsouleas, Physical mechanisms in the plasma wake-field accelerator, Phys. Rev. A 33, 2056 (1986).

[40] P. Sprangle, B. Hafizi, J. R. Peñano, R. F. Hubbard, A. Ting, C. I. Moore, D. F. Gordon, A. Zigler, D. Kaganovich, and T.M.J. Antonsen, Wakefield generation and GeV acceleration in tapered plasma channels, Phys. Rev. E 63, 056405 (2001). 
[41] S. Bulanov, V. Vshivkov, and G. Dudnikova, Fiz. Plazmy 23, 284 (1997).

[42] A. Pukhov and I. Kostyukov, Control of laser-wakefield acceleration by the plasma-density profile, Phys. Rev. E 77, 025401 (2008).

[43] W. Rittershofer, C. B. Schroeder, E. Esarey, F. J. Grüner, and W. P. Leemans, Tapered plasma channels to phase-lock accelerating and focusing forces in laser-plasma accelerators, Phys. Plasmas 17, 063104 (2010).

[44] C. DesRosiers, V. Moskvin, A. F. Bielajew, and L. Papiez, $150-250 \mathrm{MeV}$ electron beams in radiation therapy, Phys. Med. Biol. 45, 1781 (2000).

[45] C. Yeboah, G. A. Sandison, and V. Moskvin, Optimization of intensity-modulated very high energy $(50-250 \mathrm{MeV})$ electron therapy, Phys. Med. Biol. 47, 1285 (2002). 